\title{
Prognostic Factors to Predict ICU Mortality in Patients with ARDS Who Received Early and Prolonged Prone Positioning Therapy
}

\section{Po-Hsin Lee}

Taichung Veterans General Hospital

Pin-Kuei Fu ( $\nabla$ yetquen@gmail.com)

Taichung Veterans General Hospital https://orcid.org/0000-0002-9416-4094

\section{Research}

Keywords: Acute respiratory distress syndrome, Prone positioning, Prognostic factors, ICU mortality

Posted Date: March 1st, 2021

DOl: https://doi.org/10.21203/rs.3.rs-247253/v1

License: (c) (1) This work is licensed under a Creative Commons Attribution 4.0 International License.

Read Full License 


\title{
Prognostic Factors to Predict ICU Mortality in Patients with ARDS Who Received Early and Prolonged Prone Positioning Therapy
}

\author{
Po-Hsin Lee ${ }^{1 \#}$, Pin-Kuei Fu ${ }^{2-5 \# *}$
}

${ }^{1}$ Division of Chest, Department of Internal Medicine, Taichung Veterans General Hospital, Taichung, 40705, Taiwan

${ }^{2}$ Department of Critical Care Medicine, Taichung Veterans General Hospital, Taichung 407219, Taiwan

${ }^{3}$ Ph.D. Program in Translational Medicine, National Chung Hsing University, Taichung, 402010, Taiwan

${ }^{4}$ College of Human Science and Social Innovation, Hungkuang University, Taichung; 433304, Taiwan

${ }^{5}$ Department of Computer Science, Tunghai University, Taichung 407224, Taiwan Pin-Kuei Fu: https://orcid.org/0000-0002-9416-4094

\#Equal contribution of work: Po-Hsin Lee \& Pin-Kuei Fu

*Corresponding author: Dr. Pin-Kuei Fu, M.D, Ph.D. Associate Professor.

Department of Critical Care Medicine, Taichung Veterans General Hospital, 1650 Taiwan Boulevard Sect. 4, Taichung, Taiwan 40705

Tel: 886-4-2359-2525 ext 3163; Fax: 886-4-2374-1397

E-mail: yetquen@gmail.com 


\begin{abstract}
Background: Early and prolonged prone positioning (PP) could reduce the mortality in patients with moderate to severe ARDS, however, factors associated with mortality in the intensive care unit (ICU) remain unclear. The aim of this study is to identified factors associated with mortality and create the prognostic score in patients with ARDS who underwent early and prolonged PP.
\end{abstract}

Methods: This retrospective study included patients with moderate to severe ARDS admitted to the intensive care unit (ICU) from January 2015 to June 2018 in a tertiary referral center in Taiwan and who received early and prolonged PP. Demographic data, disease severity score, comorbidities, and clinical outcomes were recorded. Univariate and multivariate regression models were used to estimate the odds ratio (OR) of ICU mortality. Receiver operating characteristic (ROC) curve analysis were performed to identify the cutoff value of parameters.

Results: A total of 116 patients were enrolled. In the multivariate analysis, three factors were significantly associated with mortality: renal replacement therapy (RRT; OR: 3.38, 1.55-7.36), malignant comorbidity (OR: 7.42, 2.06-26.70), and noninfluenza-related ARDS (OR: 3.78, 1.0713.29). Age, RRT, noninfluenza-related ARDS, malignant comorbidity, and APACHE II score were included in a composite prone score, which demonstrated an area under the curve of 0.816 for predicting mortality risk. The mortality risk in ICU was $27.1 \%$ in the low-risk group (prone score: $0-2$ ) and $84.2 \%$ in the high-risk group (prone score: 3-5).

Conclusions: For patients with moderate to severe ARDS even receiving early and prolonged PP in ICU, poor prognostic factors were age, RRT, malignant comorbidity, noninfluenza-related ARDS, and higher APACHE II score. High mortality should be informed to the family of patient if their prone score was more than 3 points.

\title{
Keywords
}

Acute respiratory distress syndrome, Prone positioning, Prognostic factors, ICU mortality 


\section{Background}

The prevalence of acute respiratory distress syndrome (ARDS) in patients admitted to the intensive care units (ICU) is approximately $10 \%,[1]$ and the mortality rate ranges from $30 \%$ to $50 \%$ because of the high heterogeneity in ARDS. [2,3] Although pharmaceutical treatment is limited, prone positioning (PP) improves the outcomes of patients with ARDS. [3-5] In 1976, Piehl and Brown proposed that PP therapy could improve oxygenation in patients with ARDS. [6] Since 2001, several randomized control trials have demonstrated the survival benefit of PP therapy in patients with ARDS. [7-20] In 2013, The PROSEVA trial demonstrated that early application and prolonged duration of PP significantly reduced mortality in patients with moderate to severe ARDS. [7] Since then, five meta-analyses have recommend that in patients with ARDS requiring PP therapy, early introduction of PP accompanied by lung protective strategy and prolonged PP to $\geq 10-12 \mathrm{~h}$ per day were associated with lower mortality. [21-29]

PP has become a standard treatment in ARDS, and numerous studies have revealed factors associated with lower mortality. [4, 5, 30] However, few studies have discussed the poor prognostic factors in patients with ARDS who received early and prolonged PP therapy. [30, 31] Modrykamien et al. analyzed 43 patients with severe ARDS treated with PP and found that only three parameters were significant predictors of survival in ICU: APACHE II score, plateau pressure (Pplat), and driving pressure. [30] Kao et al. enrolled 65 patients with severe influenzarelated pulmonary ARDS and found three factors to be independently associated with 60-day mortality: pneumonia severe index, renal replacement therapy (RRT), and dynamic change in driving pressure. [31] However, these prognostic factors are of limited clinical utility in predicting which patients will benefit from PP therapy because of the lack of standardized the PP protocol, only on influenza-related ARDS, lack of consideration of ICU mortality, and lack of a scoring system for clinical application. [30, 31] In the current study, we identified factors associated ICU mortality in 116 patients with ARDS who received early and prolonged PP therapy and developed 
a prognostic score (prone score) to predict ICU mortality.

\section{Methods}

\subsection{Study design and patients}

This retrospective cohort study was conducted in the medical ICUs of Taichung Veterans General Hospital (TCVGH), a 1200-bed tertiary referral center in Taiwan, from January 2015 to June 2018. We enrolled patients diagnosed as having ARDS who received mechanical ventilation in ICUs and were treated with PP for moderate to severe hypoxemia despite a positive end-expiratory pressure (PEEP) of $>10 \mathrm{cmH}_{2} \mathrm{O}$. Moderate to severe hypoxemia was defined as a $\mathrm{PaO}_{2} /$ fraction concentration of inspired oxygen $\left(\mathrm{FiO}_{2}\right)$ ratio $<150 \mathrm{mmHg}$ according to previous clinical trials [32, 33] and the Berlin definition of ARDS. [33] We excluded patients who received PP therapy for $<6$ $\mathrm{h}$ and those who received extracorporeal membrane oxygenation due to failed PP therapy. Data related to demographics, laboratory examination, period from hypoxemia to PP, duration of PP therapy, ventilator settings, comorbidities, and clinical outcomes were extracted from the electronic medical records. The study protocol was reviewed and approved by the Institutional Review Board of TCVGH (IRB number, CE19379A; date of approval, October 25, 2019); the need for patient consent was waived due to the retrospective study design and anonymization and deidentification of patient data prior to analysis.

\subsection{Mechanical ventilator setting, recruitment maneuver, and protocol of PP therapy in medical}

\section{ICUs}

Patients diagnosed ARDS were treated with lung protective strategy to maintain Pplat $\leq 30 \mathrm{~cm}$ $\mathrm{H}_{2} \mathrm{O}$ by using lower tidal volume ventilation (goal of tidal volume: $4-8 \mathrm{~mL} / \mathrm{kg}$ predicted body weight). The setting of PEEP in our ICUs was followed by a lower PEEP strategy according to previous research and meta-analysis. [34-36] $\mathrm{FiO}_{2}$ in the ventilator was adjusted to keep oxyhemoglobin saturation by pulse oximetry $\left(\mathrm{SpO}_{2}\right)>90 \%$. The $\mathrm{PEEP}-\mathrm{FiO}_{2}$ combinations were the following: $5-8 / 0.5,8-10 / 0.6,10-12 / 0.7,12-14 / 0.8,14-16 / 0.9$, and 16-18/1.0. [37] For 
patients who failed to maintain the goal of $\mathrm{SpO}_{2} \geq 90 \%$ even using $\mathrm{FiO}_{2}$ of $>0.6$, recruitment maneuvers (RMs) were indicated through brief application of a high level of continuous positive airway pressure (CPAP) to correct hypoxemia. In our hospital, RMs include sustained inflation by abruptly raising the CPAP to $40 \mathrm{cmH}_{2} \mathrm{O}$ for $40 \mathrm{~s}$. [38, 39] $\mathrm{PP}$ was initiated as rescue therapy for patients with ARDS who experienced refractory hypoxemia within $24 \mathrm{~h}$, provided the following criteria were met: $\mathrm{PaO}_{2} / \mathrm{FiO}_{2}<150 \mathrm{mmHg}, \mathrm{FiO}_{2} \geq 0.6$, and $\mathrm{PEEP} \geq 10 \mathrm{cmH}_{2} \mathrm{O}$.

The protocol of PP therapy was according to previous publication. [37] In brief, patients lied in a prone position on a silicone pad, with their dependent parts supported by silicone cushions. Patients received PP continuously for 48-72 h and even longer until $\mathrm{PaO}_{2} / \mathrm{FiO}_{2}$ remained $<150$. During PP therapy, patients were turned right or left alternately every $2 \mathrm{~h}$ to avoid pressure sore formation. After hypoxemia improved and clinical condition stabilized (i.e., when $\mathrm{SpO}_{2}>90 \%$ and $\mathrm{FiO}_{2}<60 \%$ for $>24 \mathrm{~h}$ after at least $48-72 \mathrm{~h}$ of $\mathrm{PP}$ therapy), patients lied in the supine position.

\subsection{Data collection, assessment, and outcome measures}

Data were collected on age, sex, body mass index (BMI), Acute Physiology and Chronic Health Evaluation II (APACHE II) score, and major comorbidities. The major comorbidities were identified using the International Classification of Diseases, Ninth Revision, Clinical Modification (ICD-9-CM) code, such as congestive heart failure (CHF), coronary artery disease (CAD), interstitial lung disease (ILD), chronic obstructive pulmonary disease (COPD), diabetes mellitus (DM), chronic kidney disease (CKD), liver cirrhosis, autoimmune disease, and malignancy. Patients who received RRT during PP therapy were identified and analyzed. Parameters of ventilator settings extracted from electronic medical records included the following: mode of ventilation, tidal volume, peak inspiratory pressure (PIP), PEEP, and Pplat. The primary outcome was ICU mortality, which was defined as death in the ICU.

\subsection{Statistical analysis}


Statistical analyses and database management were performed using SPSS (version 22.0; IBM, Armonk, NY, USA). Categorical variables are presented as frequencies and percentages and were analyzed with the chi-square test. Nonparametric data were assessed using the Mann-Whitney U test and are presented as the median and interquartile range (IQR). Univariate and multivariate logistic regression models were used to estimate the odds ratio (OR). Receiver operating characteristic (ROC) curve analysis were performed for all the parameters measured and the cutoff points were decided to maximize the sum of sensitivity and specificity values of the respective ROC curves. In this study, we used the two-tailed test, and significance was set at $P<0.05$.

\section{Results}

\subsection{Patients' clinical and demographic characteristics}

From January 2015 to June 2018, 116 patients with ARDS received mechanical ventilation in ICUs and were treated with PP for moderate to severe hypoxemia despite using PEEP of $>10$ $\mathrm{cmH}_{2} \mathrm{O}$ (Figure 1). Table 1 presents the patients' demographic characteristics, etiology of ARDS, comorbidities, protocol of PP therapy, ventilator parameters, and ICU mortality (Table 1). The time from diagnosis of ARDS to initiation of PP therapy was 18.3 (IQR, 8.4-33.4) h, and the duration of continuous PP therapy was $66.1(44.4-84.5)$ h, which fit the current treatment concept of early and prolonged PP in ARDS (Table 1). In this cohort, the major cause of ARDS was noninfluenza-related ARDS $(\mathrm{n}=83,71.6 \%)$, followed by influenza-related ARDS $(\mathrm{n}=20,17.2 \%)$ and extrapulmonary ARDS $(\mathrm{n}=13,11.2 \%)$. The median APACHE II score was 31, indicating high clinical severity in this cohort. The ICU mortality was $55.2 \%(n=64)$. Figure 1 presents the details of enrollment and follow-up.

\subsection{Clinical and demographic characteristics between surviving and nonsurviving patients}

Clinicodemographic parameters were compared between surviving and nonsurviving patients (Table 2). The nonsurviving patients were older, had a malignant comorbidity, had a higher 
APACHE II score, noninfluenza-related pulmonary ARDS and received RRT more frequently (all $P<0.05)$. Other variables were not significantly different between the surviving and nonsurviving groups (Table 2).

\subsection{Factors associated with ICU mortality for patients with ARDS who received PP therapy}

Table 3 summarizes the results of logistic regression analysis for determining factors associated with ICU mortality. Univariate analysis identified five factors associated with mortality: male sex $(\mathrm{OR}, 1.04 ; 95 \% \mathrm{CI}, 1.01-1.07, P=0.003)$, APACHE II score $(\mathrm{OR}, 1.07 ; 95 \% \mathrm{CI}, 1.01-1.14 ; P=$ 0.029), noninfluenza-related pulmonary ARDS (OR, 3.78 compared with extrapulmonary ARDS; 95\% CI, 1.07-13.29; $P=0.039)$, RRT (OR, 3.38; 95\% CI, 1.55-7.36; $P=0.002)$, and active malignant disease $(\mathrm{OR}, 7.42 ; 95 \% \mathrm{CI}, 2.06-26.7 ; P=0.002)$. Multivariate analysis indicated that noninfluenza-related pulmonary ARDS (OR: 5.17 compared with extrapulmonary ARDS, 95\% CI: 1.16-23.16), RRT (OR, 4.05; 95\% CI, 1.54-10.67; $P=0.005)$, and active malignant disease (OR, 8.86; 95\% CI, 2.22-35.41; $P=0.003$ ) were significant predictive factors of ARDS-related mortality.

\subsection{Composition of prone score and its prediction of ICU mortality with PP therapy}

A "prone score" was generated to predict the risk of ICU mortality. Receiver operating curves (ROC) were plotted to identify the optimal cutoff threshold in each parameter for predicting ICU mortality (Figure 2). The cutoffs were the following: age, 53 years $(\mathrm{AUC}=0.668)$ and APACHE II score, 33 points (AUC $=0.623$ ) (Figure 2 and Table 4). The composite "prone score" to predict poor prognosis included five parameters: (1) age $\geq 53$ years, (2) APACHE II $\geq 33$, (3) receiving RRT in the ICU, (4) noninfluenza-related pulmonary ARDS, and (5) malignancy. Each item was assigned 1 point, and the total prone score ranged from 0 to 5 points. The cutoff value of the prone score with the best predictive power of ICU mortality was $\geq 3$ points $(A U C=0.816)$, which was

better than the APACHE II score (Table 4). The ICU mortality in the low-risk group (prone score: 0-2 points) was $27.1 \%$; however, the ICU mortality in the high-risk group (prone score 3-5) was 
$84.2 \%$. Univariate analysis revealed that prone score $\geq 3$ points had a significantly higher risk of ICU mortality (OR, 14.33; 95\% CI, 5.74-35.77; $P<0.001$; Table 3). As shown in Table 4, the sensitivity of prone score to predict ICU mortality was $75.0 \%$ and the specificity was $82.7 \%$.

\section{Discussion}

The current study had three major findings. First, we identified five factors associated with ICU mortality in patients with moderate to severe ARDS who received PP therapy: age, higher APACHE II score, noninfluenza-related pulmonary ARDS, RRT, and malignant comorbidity. Second, we developed a new clinical scoring tool - the prone score - to predict refractoriness to PP therapy and high risk of ICU mortality due to advanced ARDS. Third, our data revealed that the ICU mortality was $84.2 \%$ in the high-risk group (prone score $3-5$ ) and $27.1 \%$ in the low-risk group (prone score 0-2). This is the first real-world study to evaluate the treatment outcomes of PP therapy in patients with moderate to severe ARDS and develop a prediction score of ICU mortality in them.

Factors associated with good outcomes for these patients are early PP initiation, prolonged PP treatment sessions, and combinations with lung protective strategies. [7, 13] However, how early should PP therapy be initiated to reduce mortality in ARDS remains unclear. Guérin et al. (2004) proposed that PP therapy should be initiated "as early as possible" in patients with ARDS, ${ }^{7}$ which has been widely followed, with PP being initiated between 6 and $72 \mathrm{~h}$ after ARDS diagnosis. [3, 7, 9, 12, 13, 17, 18] In 2013, the PROSEVA trial clarified that PP therapy should be initiated within $36 \mathrm{~h}$ of ARDS diagnosis. ${ }^{3}$ In our medical ICUs, the protocol is PP initiation within $24 \mathrm{~h}$ of the diagnosis of moderate to severe ARDS. In the current study, the timing of PP initiation was within $36 \mathrm{~h}$ (median: $18.3 \mathrm{~h}$, IQR: 8.4-33.4). In addition, no significant difference between the surviving and nonsurviving groups regarding the timing of PP initiation was noted $(15.6$ vs. $21.3 \mathrm{~h}, P=$ 0.084). Therefore, the timing of PP therapy is less likely to be a confounder in ICU mortality in this cohort. 
Prolonged PP therapy may be a critical factor associated with mortality. $[7,9,17,18]$ In the PROSEVA trial, the goal of PP therapy was $>20$-h duration, and the actual dose was $17 \mathrm{~h}$ on average, which reduced mortality in patients with moderate to severe ARDS. [7] Two metaanalyses stated that the PP therapy $>12 \mathrm{~h}$ /day significantly reduced mortality in patients with ARDS having refractory hypoxemia. [21, 25] In our medical ICUs, patients received PP continuously for 48-72 h and even longer if $\mathrm{PaO}_{2} / \mathrm{FiO}_{2}$ persisted to be <150. [37] A recent prospective study performed $231 \mathrm{PP}$ sessions with a mean length of $21.5 \pm 5 \mathrm{~h}$ per session in patients with ARDS and recommended that the duration of PP therapy should be $\geq 24 \mathrm{~h}$, depending on whether $\mathrm{PaO}_{2} / \mathrm{FiO}_{2}$ remains <150. [40] In the current study, PP therapy was significantly longer than 20 h (median: 66.1 h, IQR: 44.4-85.4), with no significant difference between the surviving and nonsurviving groups (69.3 vs. $61.1 \mathrm{~h}, p=0.170)$. Therefore, the dose of PP therapy is less likely to be a confounder in ICU mortality in this cohort.

Lung protective strategy in conjunction with PP appeared to be a useful approach. Two metaanalyses revealed that the benefit of PP therapy in reducing mortality was only found in combination with the lung protective strategy. [21, 22] At our ICUs, the lung protective strategy is the standard of care. Therefore, in the current study, ventilation settings, including tidal volume, PEEP, and Pplat, were not significantly different between the surviving and nonsurviving groups.

The main strength of this study is the development of a scoring system to evaluate patients with moderate to severe ARDS admitted to the ICUs to determine who will receive greater benefit from early and prolonged PP therapy. Several studies have attempted to determine factors associated with mortality in patients with ARDS, including those receiving PP therapy. Lim et al. found that oxygenation improved faster in patients with extrapulmonary ARDS than pneumoniarelated ARDS. [41] Kao et al. retrospectively examined 65 patients with influenza-related ARDS treated with PP and found that 60-day mortality was associated with a higher pneumonia severity index, RRT, and increased dynamic driving pressure. [31] Modrykamien et al. retrospectively 
studied 43 patients and identified only three factors-APACHE II score, Pplat, and driving pressure - to be associated with mortality when receiving PP therapy. [30] These studies provided some clues of mortality predictors but were limited in developing a scoring system. In our study, we identified five prognostic factors from 116 patients and used their cutoffs to develop the prone score to predict ICU mortality: age $\geq 53$ years, APACHE II score $\geq 33$ points, receiving RRT, malignant comorbidity, and noninfluenza-related ARDS.

This study has several limitations. First, because of the retrospective design, heterogeneity may have existed in each patient. Second, the study was conducted at medical ICUs in a single medical center rather than a multicenter study, meaning that the results may not be generalizable. In practice, the protocol of PP therapy, especially timing of initiation and dosage, and combination with other intensive respiratory therapies, such as recruitment maneuver and fluid strategy, vary between ICUs in different hospitals. In our medical ICUs, we followed a standard protocol of early and prolonged PP therapy since 2007.[37] We treated patients with sepsis, pneumonia, and ARDS by using the documented protocol modified from the latest severe sepsis guideline.[42] In addition, our ICUs were serviced by full-time intensivists. Therefore, heterogeneity in ICU care and PP therapy protocols was minimal in the present study. Third, critically ill patients in different countries or ICUs may have different demographic patterns, disease severity, and comorbidities, which can confound ICU mortality. Finally, our results may not be generalizable to patients with ARDS in pediatric, neurosurgical, surgical, and cardiac ICUs, because the current study included only adult patients admitted to the medical ICUs in TCVGH.

\section{Conclusions}

We developed the prone score to predict ICU mortality in patients with ARDS receiving early and prolonged PP therapy. The prone score comprises five parameters: age $\geq 53$ years, receiving RRT in ICU, noninfluenza-related pulmonary ARDS, malignancy, and APACHE II score $\geq 33$. The corresponding mortality rates for low risk (score 0-2) and high risk (score 3-5) were $27.1 \%$ 
and $84.2 \%$, respectively. To the best of our knowledge, the current study is the first article to develop the prognostic score for patients with ARDS receiving early and prolonged PP therapy.

\section{List of abbreviations}

ARDS: Acute respiratory distress syndrome

APACHE II: Acute Physiology and Chronic Health Evaluation II

AUC: Area under the receiver operating characteristic curve

CAD: Coronary artery disease

CHF: Congestive heart failure

CKD: Chronic kidney disease

COPD: Chronic obstructive pulmonary disease

DM: Diabetes Mellitus

ECMO: extracorporeal membrane oxygenation

ICU: Intensive care unit

ILD: Interstitial lung disease

PEEP: Positive end-expiratory pressure

PIP: Peak inspiratory pressure

Pplat: Plateau pressure

ROC: Receiver operating curves

\section{Declarations}

Ethics approval and consent to participate: The study protocol was reviewed and approved by the Institutional Review Board of TCVGH (IRB number, CE19379A; date of approval, October $25,2019)$; the need for patient consent was waived due to the retrospective study design and anonymization and deidentification of patient data prior to analysis.

Consent for publication: Not applicable 
Availability of data and materials: The datasets generated and/or analysed during the current study are not publicly available due to this study was conducted by using deidentified medical records from TCVGH but are available from the corresponding author on reasonable request. Competing interests: The authors declare that they have no competing interests.

Funding: The authors sincerely appreciate funding in part by the Department of Medical Research of Taichung Veterans General Hospital (TCVGH-1094404C) and the Ministry of Science and Technology (Taiwan) (MOST 109-2410-H-075A-001-SSS).

Author contributions : Conceptualization, P.-K.F; Data curation, P.-H.L and P.-K.F; Formal analysis, P.-H.L and P.-K.F; Writing - original draft, P.-H.L and P.-K.F; Writing - review \& editing, P.-H.L and P.-K.F. All authors read and approved the final manuscript. Acknowledgments: We thank Miss Chiann-Yi Hsu from the Biostatistics Task Force of Taichung Veterans General Hospital for her assistance. This manuscript was edited by Wallace Academic Editing. 


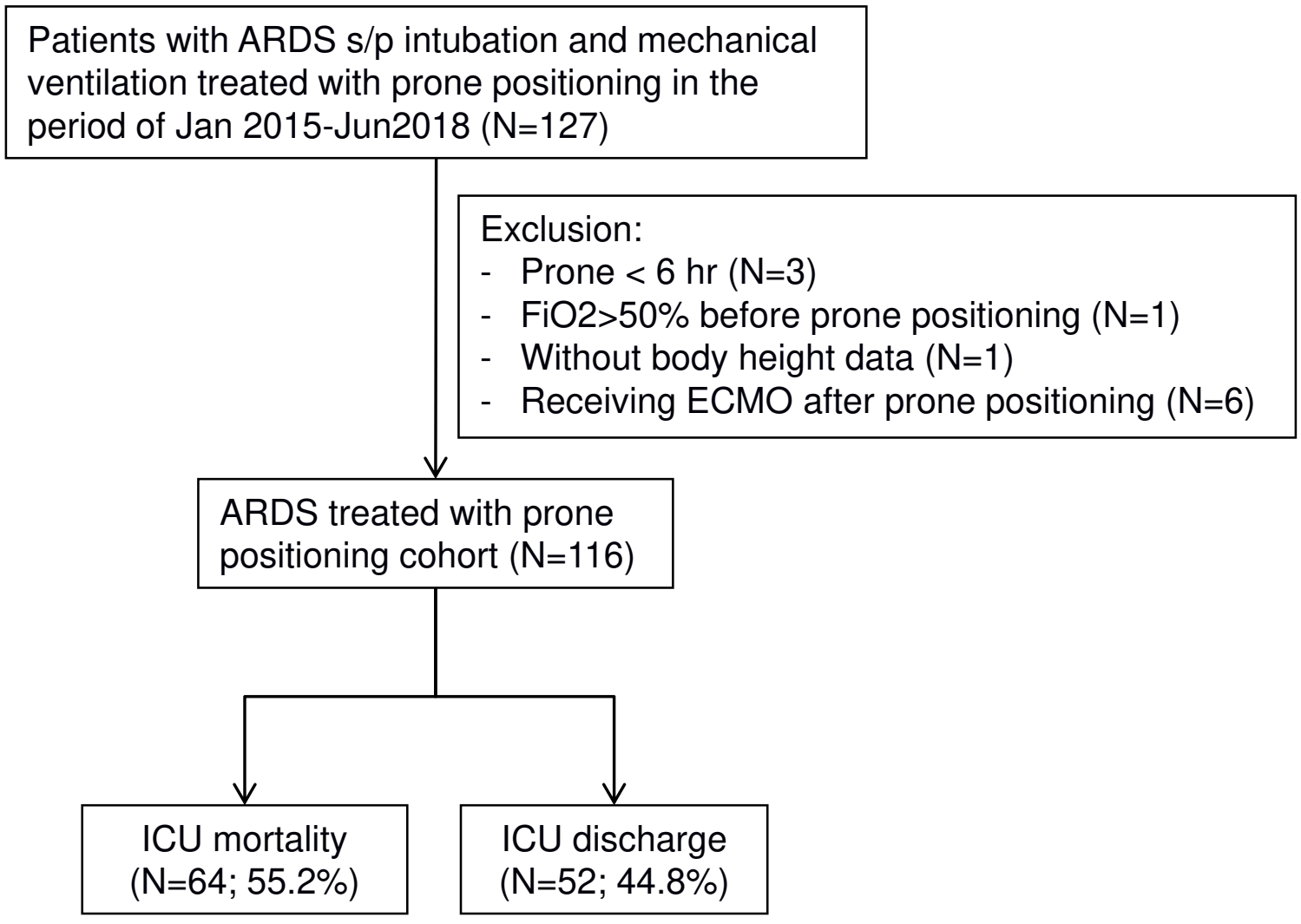

Fig. 1 Enrollment and follow-up of the study participants. ARDS, acute respiratory distress syndrome; FiO2, fraction concentration of inspired oxygen; ECMO, extracorporeal membrane oxygenation; ICU: intensive care unit 


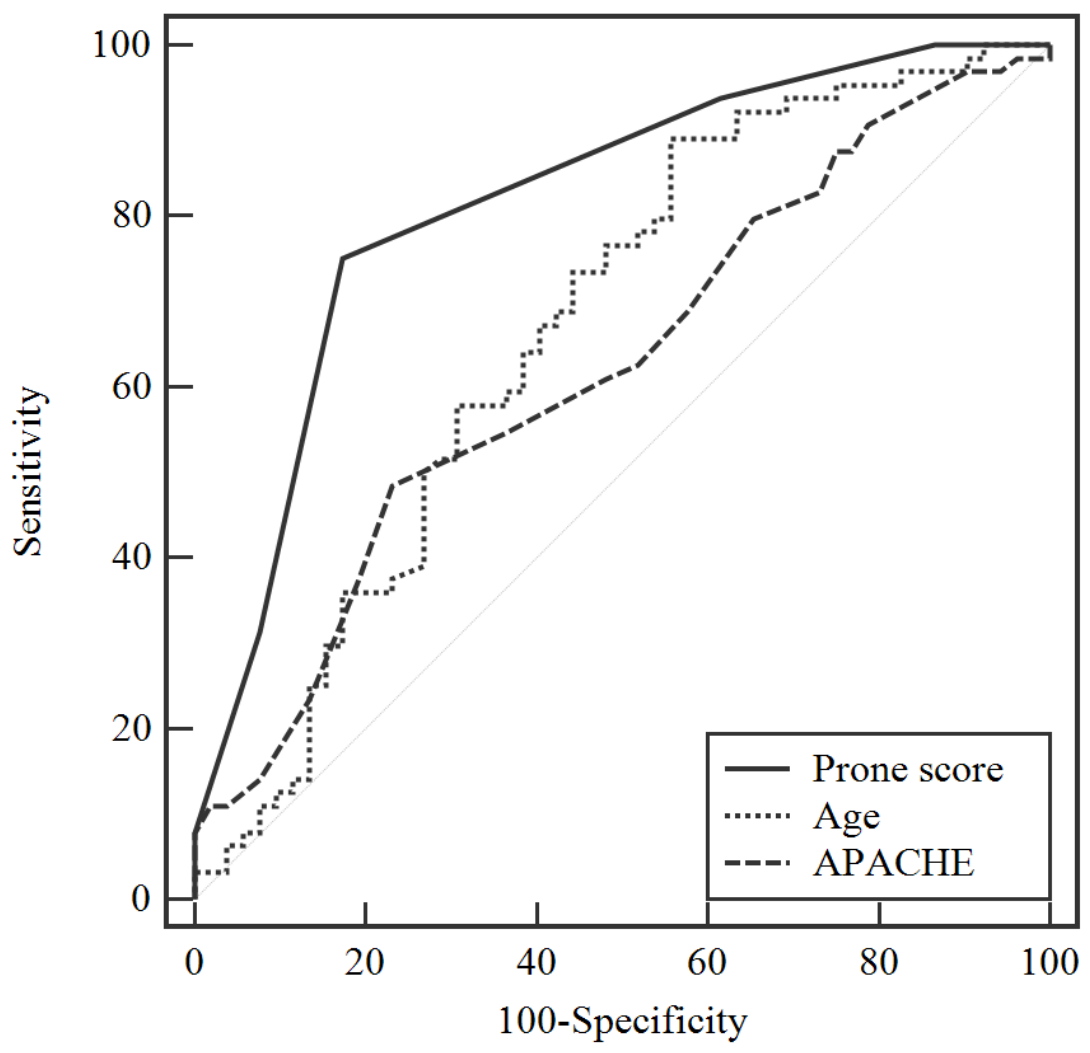

Fig. 2 Receiver operating curves analysis of age, APACHE II score, and prone score for predicting ICU mortality in patients with acute respiratory distress syndrome receiving early and prolonged prone positioning. 
Table 1 Patients' clinicodemographic characteristics

\begin{tabular}{|c|c|c|}
\hline \multirow{2}{*}{ Demographic data } & \multicolumn{2}{|c|}{ Total $(n=116)$} \\
\hline & & \\
\hline Age & 62.9 & $(52.1-74.2)$ \\
\hline Gender-Male & 70 & $(60.3 \%)$ \\
\hline $\mathrm{BMI}\left(\mathrm{kg} / \mathrm{m}^{2}\right)$ & 24.4 & $(21.9-27.9)$ \\
\hline APACHE II score & 31 & $(27-35)$ \\
\hline ICU mortality & 64 & $(55.2 \%)$ \\
\hline \multicolumn{3}{|l|}{ Cause of ARDS } \\
\hline Non-influenza pulmonary ARDS & 83 & $(71.6 \%)$ \\
\hline Influenza & 20 & $(17.2 \%)$ \\
\hline Extrapulmonary ARDS & 13 & $(11.2 \%)$ \\
\hline Renal replacement therapy & 52 & $(44.8 \%)$ \\
\hline \multicolumn{3}{|l|}{ Comorbidity } \\
\hline $\mathrm{CHF}$ & 6 & $(5.2 \%)$ \\
\hline CAD & 8 & $(6.9 \%)$ \\
\hline ILD & 6 & $(5.2 \%)$ \\
\hline COPD & 10 & $(8.6 \%)$ \\
\hline DM & 40 & $(34.5 \%)$ \\
\hline CKD & 44 & $(37.9 \%)$ \\
\hline Liver Cirrhosis & 10 & $(8.6 \%)$ \\
\hline Autoimmune disease & 18 & $(15.5 \%)$ \\
\hline Malignancy & 23 & $(19.8 \%)$ \\
\hline \multicolumn{3}{|l|}{ Prone information } \\
\hline Timing from ARDS to prone $(\mathrm{h})$ & 18.3 & $(8.4-33.4)$ \\
\hline Total prone duration $(\mathrm{h})$ & 66.1 & $(44.4-84.5)$ \\
\hline $\mathrm{PF}$ ratio & 90.6 & $(71.0-113.8)$ \\
\hline \multicolumn{3}{|l|}{ Ventilation setting } \\
\hline Mode, volume control & 114 & $(95.7 \%)$ \\
\hline Tidal volume (ml/Kg) & 6.0 & $(5.7-6.5)$ \\
\hline PEEP $(\mathrm{mmHg})$ & 14.0 & $(14-16)$ \\
\hline PIP (mmHg) & 32.0 & $(29-35)$ \\
\hline Pplat (mmHg) & 29.0 & $(26.2-31)$ \\
\hline Driving Pressure (mmHg) & 13.0 & $(11.8-16.2)$ \\
\hline Compliance (ml/mmHg) & 25.8 & $(21.3-32.5)$ \\
\hline
\end{tabular}

Continuous data are expressed as median and IQR. Categorical data were expressed number and percentage. CHF, congestive heart failure; CAD, coronary artery disease; ILD, interstitial lung disease; COPD, chronic obstructive pulmonary disease, DM, diabetes mellitus; CKD, chronic kidney disease; PEEP: positive end-expiratory pressure ; PIP, peak inspiratory pressure; Pplat, plateau pressure 
Table 2 Characteristics of survivors and nonsurvivors in patients with ARDS receiving prone positioning

\begin{tabular}{|c|c|c|c|c|c|}
\hline \multirow{2}{*}{ Demographic data } & \multicolumn{2}{|c|}{ Alive $(\mathrm{n}=52)$} & \multicolumn{2}{|c|}{ Death $(n=64)$} & \multirow[t]{2}{*}{$p$ value } \\
\hline & & & & & \\
\hline Age & 56.7 & $(46.2-68.3)$ & 65.7 & $(57.3-76.0)$ & $0.002 * *$ \\
\hline Gender-Male & 34 & $(65.4 \%)$ & 36 & $(56.3 \%)$ & 0.345 \\
\hline BMI $\left(\mathrm{kg} / \mathrm{m}^{2}\right)$ & 25.5 & $(22.0-28.3)$ & 24.0 & $(21.8-27.1)$ & 0.289 \\
\hline APACHE II score & 30.0 & $(25.8-32.0)$ & 32.0 & $(28-35)$ & $0.022 *$ \\
\hline Cause of ARDS & & & & & $0.028^{*}$ \\
\hline Non-influenza pulmonary ARDS & 31 & $(59.6 \%)$ & 52 & $(81.3 \%)$ & \\
\hline Influenza & 12 & $(23.1 \%)$ & 8 & $(12.5 \%)$ & \\
\hline Extrapulmonary ARDS & 9 & $(17.3 \%)$ & 4 & $(6.3 \%)$ & \\
\hline Renal replacement therapy & 15 & $(28.8 \%)$ & 37 & $(57.8 \%)$ & $0.003 * *$ \\
\hline \multicolumn{6}{|l|}{ Comorbidity } \\
\hline $\mathrm{CHF}^{\mathrm{f}}$ & 4 & $(7.7 \%)$ & 2 & $(3.1 \%)$ & 0.406 \\
\hline $\mathrm{CAD}^{\mathrm{f}}$ & 4 & $(7.7 \%)$ & 4 & $(6.3 \%)$ & 1.000 \\
\hline $\operatorname{ILD}^{\mathrm{f}}$ & 3 & $(5.8 \%)$ & 3 & $(4.7 \%)$ & 1.000 \\
\hline $\operatorname{COPD}^{\mathrm{f}}$ & 6 & $(11.5 \%)$ & 4 & $(6.3 \%)$ & 0.340 \\
\hline $\mathrm{DM}$ & 22 & $(42.3 \%)$ & 18 & $(28.1 \%)$ & 0.121 \\
\hline CKD & 17 & $(32.7 \%)$ & 27 & $(42.2 \%)$ & 0.339 \\
\hline Cirrhosis ${ }^{f}$ & 3 & $(5.8 \%)$ & 7 & $(10.9 \%)$ & 0.508 \\
\hline Autoimmune disease & 7 & $(13.5 \%)$ & 11 & $(17.2 \%)$ & 0.617 \\
\hline Malignancy & 3 & $(5.8 \%)$ & 20 & $(31.3 \%)$ & $0.001 * *$ \\
\hline \multicolumn{6}{|l|}{ Prone information } \\
\hline Timing from ARDS to prone (h) & 15.6 & $(7.6-30.2)$ & 21.3 & $(9.1-46.8)$ & 0.084 \\
\hline Total prone duration $(\mathrm{h})$ & 69.3 & $(52.8-84.2)$ & 61.1 & $(36.8-84.5)$ & 0.170 \\
\hline $\mathrm{PF}$ ratio & 93.4 & $(69.8-118.9)$ & 88.5 & $(72.2-112.4)$ & 0.363 \\
\hline \multicolumn{6}{|l|}{ Ventilation setting } \\
\hline Mode, volume control & 51 & $(98.1 \%)$ & 60 & $(93.7 \%)$ & 0.378 \\
\hline Tidal volume (ml/Kg) & 5.9 & $(5.7-6.2)$ & 6.2 & $(5.8-6.6)$ & 0.088 \\
\hline PEEP $(\mathrm{mmHg})$ & 16.0 & $(14-16)$ & 14.0 & $(14-16)$ & 0.108 \\
\hline PIP (mmHg) & 32.0 & $(29.2-35.0)$ & 32.5 & $(29-35)$ & 0.953 \\
\hline Pplat (mmHg) & 28.0 & $(26.8-30.3)$ & 29.0 & $(26-31)$ & 0.765 \\
\hline Driving Pressure (mmHg) & 13.0 & $(11-15.5)$ & 14.0 & $(12-16.2)$ & 0.349 \\
\hline Compliance $(\mathrm{ml} / \mathrm{mmHg})$ & 26.4 & $(22.8-33.5)$ & 25.4 & $(20-30.8)$ & 0.252 \\
\hline
\end{tabular}

Mann-Whitney U test. Chi-square test. ${ }^{\mathrm{f}}$ Fisher's exact test. $* p<0.05$, $* * p<0.01$. Continuous data are expressed as median and IQR. Categorical data are expressed number and percentage.

$\mathrm{CHF}$, congestive heart failure; CAD, coronary artery disease; ILD, interstitial lung disease; COPD, chronic obstructive pulmonary disease, DM, diabetes mellitus; $\mathrm{CKD}$, chronic kidney disease;

PEEP: positive end-expiratory pressure; PIP, peak inspiratory pressure; Pplat, plateau pressure 
Table 3 Logistic regression analysis of clinical variables associated with ICU mortality in patients with ARDS receiving early and prolonged prone positioning

\begin{tabular}{|c|c|c|c|c|c|c|}
\hline & \multicolumn{3}{|c|}{ Univariate analysis } & \multicolumn{3}{|c|}{ Multivariable analysis } \\
\hline & OR & $(95 \% \mathrm{CI})$ & $\mathrm{p}$ value & OR & $(95 \% \mathrm{CI})$ & $\mathrm{p}$ value \\
\hline \multicolumn{7}{|l|}{ Demographic data } \\
\hline Age & 1.04 & $(1.01-1.07)$ & $0.003 * *$ & 1.02 & $(0.99-1.05)$ & 0.139 \\
\hline Gender-Male & 0.68 & $(0.32-1.45)$ & 0.318 & & & \\
\hline BMI (kg/m2) & 0.97 & $(0.90-1.05)$ & 0.404 & & & \\
\hline APACHE II score & 1.07 & $(1.01-1.14)$ & $0.029 *$ & 1.05 & $(0.97-1.14)$ & 0.206 \\
\hline \multicolumn{7}{|l|}{ Cause of ARDS } \\
\hline Extrapulmonary ARDS & ref. & & & ref. & & \\
\hline Non-influenza pulmonary ARDS & 3.78 & $(1.07-13.29)$ & $0.039^{*}$ & 5.17 & $(1.16-23.16)$ & $0.032 *$ \\
\hline Influenza & 1.50 & $(0.34-6.58)$ & 0.591 & 2.00 & $(0.36-11.12)$ & 0.428 \\
\hline Renal replacement therapy & 3.38 & $(1.55-7.36)$ & $0.002 * *$ & 4.05 & $(1.54-10.67)$ & $0.005 * *$ \\
\hline \multicolumn{7}{|l|}{ Comorbidity } \\
\hline $\mathrm{CHF}$ & 0.39 & $(0.07-2.20)$ & 0.285 & & & \\
\hline CAD & 0.80 & $(0.19-3.37)$ & 0.761 & & & \\
\hline ILD & 0.79 & $(0.19-3.33)$ & 0.748 & & & \\
\hline COPD & 0.51 & $(0.14-1.92)$ & 0.320 & & & \\
\hline DM & 0.53 & $(0.25-1.16)$ & 0.112 & & & \\
\hline CKD & 1.50 & $(0.70-3.22)$ & 0.296 & & & \\
\hline Cirrhosis & 2.01 & $(0.49-8.18)$ & 0.332 & & & \\
\hline Autoimmune disease & 1.33 & $(0.48-3.73)$ & 0.582 & & & \\
\hline Malignancy & 7.42 & $(2.06-26.70)$ & $0.002 * *$ & 8.86 & $(2.22-35.41)$ & $0.003 * *$ \\
\hline \multicolumn{7}{|l|}{ Prone information } \\
\hline Timing from ARDS to prone (h) & 1.01 & $(1.00-1.03)$ & 0.057 & & & \\
\hline Total prone duration $(\mathrm{h})$ & 1.00 & $(0.99-1.01)$ & 0.995 & & & \\
\hline $\mathrm{PF}$ ratio & 0.99 & $(0.98-1.00)$ & 0.238 & & & \\
\hline \multicolumn{7}{|l|}{ Ventilation setting } \\
\hline Mode, pressure control & 3.40 & $(0.37-31.40)$ & 0.281 & & & \\
\hline Tidal volume (ml/Kg) & 1.42 & $(0.95-2.11)$ & 0.084 & & & \\
\hline PEEP (mmHg) & 0.93 & $(0.81-1.06)$ & 0.257 & & & \\
\hline PIP (mmHg) & 0.99 & $(0.90-1.09)$ & 0.826 & & & \\
\hline Pplat (mmHg) & 0.99 & $(0.90-1.10)$ & 0.880 & & & \\
\hline Driving Pressure (mmHg) & 1.04 & $(0.94-1.15)$ & 0.447 & & & \\
\hline Compliance (ml/mmHg) & 0.98 & $(0.94-1.02)$ & 0.376 & & & \\
\hline Prone score high risk (score 3-5) & 14.33 & $(5.74-35.77)$ & $<0.001 * *$ & & & \\
\hline
\end{tabular}

Logistic regression. $* P<0.05, * * P<0.01$. CHF, congestive heart failure; CAD, coronary artery disease; ILD, interstitial lung disease; COPD, chronic obstructive pulmonary disease, DM, diabetes mellitus; CKD, chronic kidney disease; PEEP: positive end-expiratory pressure; PIP, peak inspiratory pressure; Pplat, plateau pressure. 
Table 4. ROC curve analysis of parameters associated with ICU mortality in patients with severe ARDS receiving early and prolonged prone positioning

\begin{tabular}{lccccccccc}
\hline & AUC & $(95 \%$ CI $)$ & $p$ value & Cut-off point & Sensitivity & Specificity & Accuracy & PPV & NPV \\
\hline Prone score & 0.816 & $(0.773-0.882)$ & $<0.0001$ & $\geq 3$ & 75.00 & 82.69 & 78.45 & 84.21 & 72.88 \\
Age & 0.668 & $(0.574-0.752)$ & $0.001 * *$ & $\geq 53$ & 89.06 & 44.23 & 68.97 & 66.28 & 76.67 \\
APACHE & 0.623 & $(0.529-0.712)$ & $0.018^{*}$ & $\geq 33$ & 48.44 & 76.92 & 61.21 & 72.09 & 54.79 \\
\hline
\end{tabular}


References

1. Bellani G, Laffey JG, Pham T, Fan E, Brochard L, Esteban A, Gattinoni L, van Haren F, Larsson A, McAuley DF et al: Epidemiology, Patterns of Care, and Mortality for Patients With Acute Respiratory Distress Syndrome in Intensive Care Units in 50 Countries. JAMA 2016, 315(8):788-800.

2. Bos LD, Martin-Loeches I, Schultz MJ: ARDS: challenges in patient care and frontiers in research. Eur Respir Rev 2018, 27(147).

3. Rilinger J, Zotzmann V, Bemtgen X, Schumacher C, Biever PM, Duerschmied D, Kaier K, Stachon $\mathrm{P}$, von Zur Muhlen $\mathrm{C}$, Zehender $\mathrm{M}$ et al: Prone positioning in severe ARDS requiring extracorporeal membrane oxygenation. Crit Care 2020, 24(1):397.

4. Howell MD, Davis AM: Management of ARDS in Adults. JAMA 2018, 319(7):711-712.

5. Scholten EL, Beitler JR, Prisk GK, Malhotra A: Treatment of ARDS With Prone Positioning. Chest 2017, 151(1):215-224.

6. Piehl MA, Brown RS: Use of extreme position changes in acute respiratory failure. Crit Care Med 1976, 4(1):13-14.

7. Guerin C, Reignier J, Richard JC, Beuret P, Gacouin A, Boulain T, Mercier E, Badet M, Mercat $A$, Baudin $O$ et al: Prone positioning in severe acute respiratory distress syndrome. N Engl J Med 2013, 368(23):2159-2168.

8. Demory D, Michelet P, Arnal JM, Donati S, Forel JM, Gainnier M, Bregeon F, Papazian L: High-frequency oscillatory ventilation following prone positioning prevents a further impairment in oxygenation. Crit Care Med 2007, 35(1):106-111.

9. Mancebo J, Fernandez R, Blanch L, Rialp G, Gordo F, Ferrer M, Rodriguez F, Garro P, Ricart $P$, Vallverdu I et al: A multicenter trial of prolonged prone ventilation in severe acute respiratory distress syndrome. Am J Respir Crit Care Med 2006, 173(11):1233-1239.

10. Voggenreiter G, Aufmkolk M, Stiletto RJ, Baacke MG, Waydhas C, Ose C, Bock E, Gotzen L, Obertacke $U$, Nast-Kolb D: Prone positioning improves oxygenation in post-traumatic lung injury--a prospective randomized trial. J Trauma 2005, 59(2):333-341; discussion 341-333.

11. Papazian L, Gainnier M, Marin V, Donati S, Arnal JM, Demory D, Roch A, Forel JM, Bongrand $\mathrm{P}$, Bregeon $\mathrm{F}$ et al: Comparison of prone positioning and high-frequency oscillatory ventilation in patients with acute respiratory distress syndrome. Crit Care Med 2005, 33(10):2162-2171.

12. Curley MA, Hibberd PL, Fineman LD, Wypij D, Shih MC, Thompson JE, Grant MJ, Barr FE, Cvijanovich NZ, Sorce L et al: Effect of prone positioning on clinical outcomes in children with acute lung injury: a randomized controlled trial. JAMA 2005, 294(2):229-237.

13. Guerin C, Gaillard S, Lemasson S, Ayzac L, Girard R, Beuret P, Palmier B, Le QV, Sirodot M, Rosselli $\mathrm{S}$ et al: Effects of systematic prone positioning in hypoxemic acute respiratory failure: a randomized controlled trial. JAMA 2004, 292(19):2379-2387.

14. Watanabe I, Fujihara H, Sato K, Honda T, Ohashi S, Endoh H, Yamakura T, Taga K, Shimoji $\mathrm{K}$ : Beneficial effect of a prone position for patients with hypoxemia after transthoracic esophagectomy. Crit Care Med 2002, 30(8):1799-1802.

15. Beuret P, Carton MJ, Nourdine K, Kaaki M, Tramoni G, Ducreux JC: Prone position as prevention of lung injury in comatose patients: a prospective, randomized, controlled study. Intensive Care Med 2002, 28(5):564-569. 
16. Gattinoni L, Tognoni G, Pesenti A, Taccone P, Mascheroni D, Labarta V, Malacrida R, Di Giulio $P$, Fumagalli $R$, Pelosi $P$ et al: Effect of prone positioning on the survival of patients with acute respiratory failure. N Engl J Med 2001, 345(8):568-573.

17. Taccone P, Pesenti A, Latini R, Polli F, Vagginelli F, Mietto C, Caspani L, Raimondi F, Bordone $\mathrm{G}$, lapichino $\mathrm{G}$ et al: Prone positioning in patients with moderate and severe acute respiratory distress syndrome: a randomized controlled trial. JAMA 2009, 302(18):1977-1984.

18. Fernandez R, Trenchs X, Klamburg J, Castedo J, Serrano JM, Besso G, Tirapu JP, Santos A, Mas A, Parraga $M$ et al: Prone positioning in acute respiratory distress syndrome: a multicenter randomized clinical trial. Intensive Care Med 2008, 34(8):1487-1491.

19. Guérin C, Reignier J, Richard J-C, Beuret P, Gacouin A, Boulain T, Mercier E, Badet M, Mercat $A$, Baudin $O$ : Prone positioning in severe acute respiratory distress syndrome. New England Journal of Medicine 2013, 368(23):2159-2168.

20. Gattinoni L, Tognoni G, Pesenti A, Taccone P, Mascheroni D, Labarta V, Malacrida R, Di Giulio $P$, Fumagalli $R$, Pelosi $P$ et al: Effect of prone positioning on the survival of patients with acute respiratory failure. N Engl J Med 2001, 345(8):568-573.

21. Munshi L, Del Sorbo L, Adhikari NKJ, Hodgson CL, Wunsch H, Meade MO, Uleryk E, Mancebo J, Pesenti A, Ranieri VM et al: Prone Position for Acute Respiratory Distress Syndrome. A Systematic Review and Meta-Analysis. Ann Am Thorac Soc 2017, 14(Supplement_4):S280-S288.

22. Park SY, Kim HJ, Yoo KH, Park YB, Kim SW, Lee SJ, Kim EK, Kim JH, Kim YH, Moon JY et al: The efficacy and safety of prone positioning in adults patients with acute respiratory distress syndrome: a meta-analysis of randomized controlled trials. J Thorac Dis 2015, 7(3):356-367.

23. Sud S, Friedrich JO, Adhikari NK, Taccone P, Mancebo J, Polli F, Latini R, Pesenti A, Curley $M A$, Fernandez $R$ et al: Effect of prone positioning during mechanical ventilation on mortality among patients with acute respiratory distress syndrome: a systematic review and meta-analysis. CMAJ 2014, 186(10):E381-390.

24. Lee JM, Bae W, Lee YJ, Cho YJ: The efficacy and safety of prone positional ventilation in acute respiratory distress syndrome: updated study-level meta-analysis of $\mathbf{1 1}$ randomized controlled trials. Crit Care Med 2014, 42(5):1252-1262.

25. Beitler JR, Shaefi S, Montesi SB, Devlin A, Loring SH, Talmor D, Malhotra A: Prone positioning reduces mortality from acute respiratory distress syndrome in the low tidal volume era: a meta-analysis. Intensive Care Med 2014, 40(3):332-341.

26. Park SY, Kim HJ, Yoo KH, Park YB, Kim SW, Lee SJ, Kim EK, Kim JH, Kim YH, Moon J-y: The efficacy and safety of prone positioning in adults patients with acute respiratory distress syndrome: a meta-analysis of randomized controlled trials. Journal of thoracic disease 2015, 7(3):356.

27. Sud S, Friedrich JO, Adhikari NK, Taccone P, Mancebo J, Polli F, Latini R, Pesenti A, Curley $M A$, Fernandez R: Effect of prone positioning during mechanical ventilation on mortality among patients with acute respiratory distress syndrome: a systematic review and meta-analysis. Canadian Medical Association Journal 2014:cmaj. 140081.

28. Lee JM, Bae W, Lee YJ, Cho Y-J: The efficacy and safety of prone positional ventilation in acute respiratory distress syndrome: updated study-level meta-analysis of 11 randomized controlled trials. Critical care medicine 2014, 42(5):1252-1262. 
29. Beitler JR, Shaefi S, Montesi SB, Devlin A, Loring SH, Talmor D, Malhotra A: Prone positioning reduces mortality from acute respiratory distress syndrome in the low tidal volume era: a meta-analysis. Intensive care medicine 2014, 40(3):332-341.

30. Modrykamien AM, Daoud Y: Factors among patients receiving prone positioning for the acute respiratory distress syndrome found useful for predicting mortality in the intensive care unit. Proc (Bayl Univ Med Cent) 2018, 31(1):1-5.

31. Kao KC, Chang KW, Chan MC, Liang SJ, Chien YC, Hu HC, Chiu LC, Chen WC, Fang WF, Chen $Y M$ et al: Predictors of survival in patients with influenza pneumonia-related severe acute respiratory distress syndrome treated with prone positioning. Ann Intensive Care 2018, 8(1):94.

32. Girard R, Baboi L, Ayzac L, Richard JC, Guerin C, Proseva trial g: The impact of patient positioning on pressure ulcers in patients with severe ARDS: results from a multicentre randomised controlled trial on prone positioning. Intensive Care Med 2014, 40(3):397403.

33. Force ADT, Ranieri VM, Rubenfeld GD, Thompson BT, Ferguson ND, Caldwell E, Fan E, Camporota L, Slutsky AS: Acute respiratory distress syndrome: the Berlin Definition. JAMA 2012, 307(23):2526-2533.

34. Brower RG, Lanken PN, MacIntyre N, Matthay MA, Morris A, Ancukiewicz M, Schoenfeld $D$, Thompson BT, National Heart L, Blood Institute ACTN: Higher versus lower positive end-expiratory pressures in patients with the acute respiratory distress syndrome. $N$ Engl J Med 2004, 351(4):327-336.

35. Acute Respiratory Distress Syndrome N, Brower RG, Matthay MA, Morris A, Schoenfeld D, Thompson BT, Wheeler A: Ventilation with lower tidal volumes as compared with traditional tidal volumes for acute lung injury and the acute respiratory distress syndrome. N Engl J Med 2000, 342(18):1301-1308.

36. Walkey AJ, Del Sorbo L, Hodgson CL, Adhikari NKJ, Wunsch H, Meade MO, Uleryk E, Hess D, Talmor DS, Thompson BT et al: Higher PEEP versus Lower PEEP Strategies for Patients with Acute Respiratory Distress Syndrome. A Systematic Review and Meta-Analysis. Ann Am Thorac Soc 2017, 14(Supplement_4):S297-S303.

37. Chan MC, Hsu JY, Liu HH, Lee YL, Pong SC, Chang LY, Kuo BI, Wu CL: Effects of prone position on inflammatory markers in patients with ARDS due to community-acquired pneumonia. J Formos Med Assoc 2007, 106(9):708-716.

38. Oczenski W, Hormann C, Keller C, Lorenzl N, Kepka A, Schwarz S, Fitzgerald RD: Recruitment maneuvers during prone positioning in patients with acute respiratory distress syndrome. Crit Care Med 2005, 33(1):54-61; quiz 62.

39. Grasso S, Terragni P, Mascia L, Fanelli V, Quintel M, Herrmann P, Hedenstierna G, Slutsky AS, Ranieri VM: Airway pressure-time curve profile (stress index) detects tidal recruitment/hyperinflation in experimental acute lung injury. Crit Care Med 2004, 32(4):1018-1027.

40. Jochmans S, Mazerand S, Chelly J, Pourcine F, Sy O, Thieulot-Rolin N, Ellrodt O, Mercier Des Rochettes E, Michaud G, Serbource-Goguel J et al: Duration of prone position sessions: a prospective cohort study. Ann Intensive Care 2020, 10(1):66.

41. Lim CM, Kim EK, Lee JS, Shim TS, Lee SD, Koh Y, Kim WS, Kim DS, Kim WD: Comparison of the response to the prone position between pulmonary and extrapulmonary acute respiratory distress syndrome. Intensive Care Med 2001, 27(3):477-485. 
42. Rhodes A, Evans LE, Alhazzani W, Levy MM, Antonelli M, Ferrer R, Kumar A, Sevransky JE, Sprung CL, Nunnally ME et al: Surviving Sepsis Campaign: International Guidelines for Management of Sepsis and Septic Shock: 2016. Crit Care Med 2017, 45(3):486-552. 
Figures

\section{Patients with ARDS s/p intubation and mechanical ventilation treated with prone positioning in the period of Jan 2015-Jun2018 ( $N=127)$}

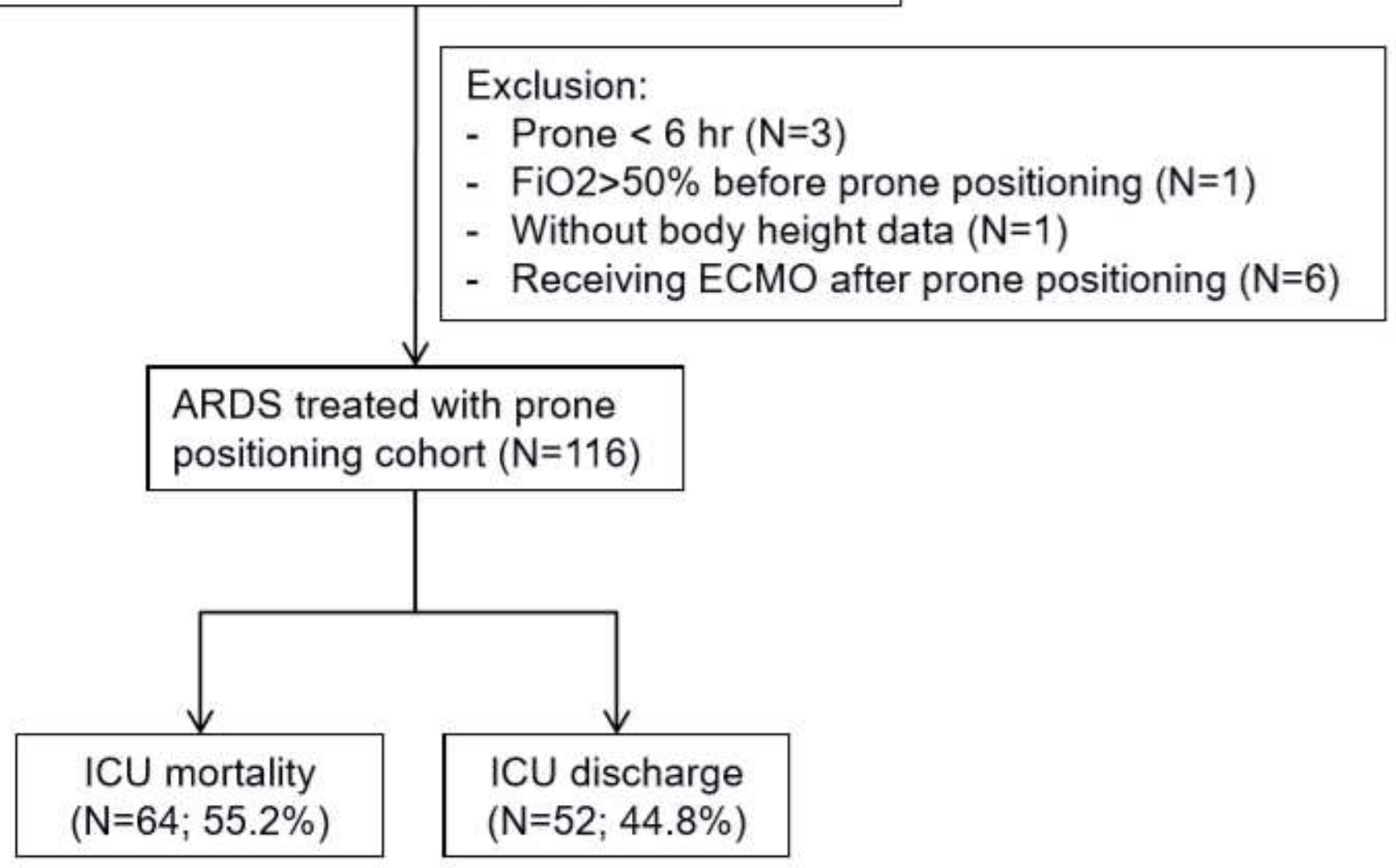

Figure 1

Enrollment and follow-up of the study participants. ARDS, acute respiratory distress syndrome; FiO2, fraction concentration of inspired oxygen; ECMO, extracorporeal membrane oxygenation; ICU: intensive care unit 


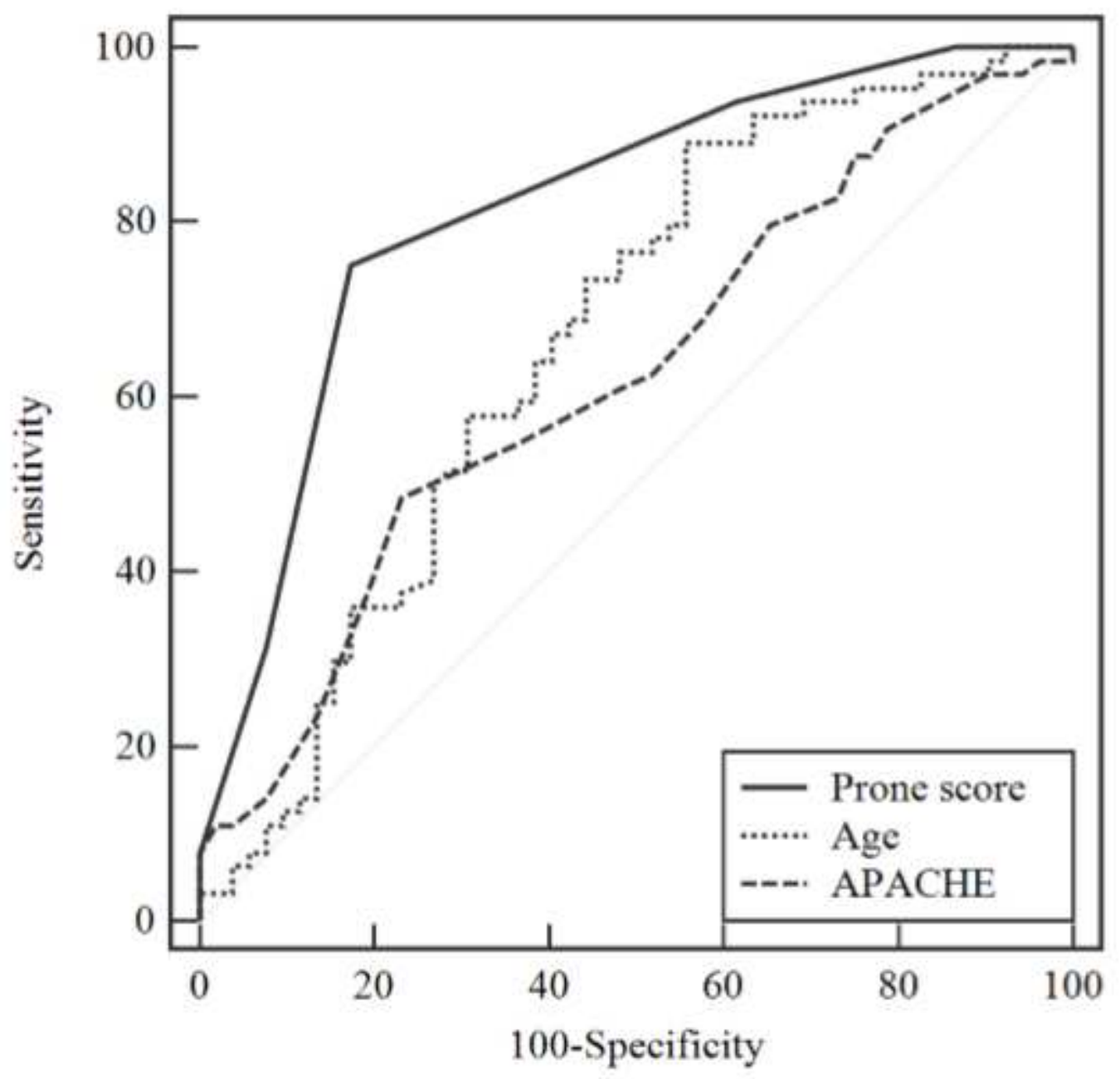

Figure 2

Receiver operating curves analysis of age, APACHE II score, and prone score for predicting ICU mortality in patients with acute respiratory distress syndrome receiving early and prolonged prone positioning. 